\title{
Exclusive photoproduction of hard dijets and magnetic susceptibility of QCD vacuum
}

\author{
V. M. Braun ${ }^{1}$, S. Gottwald ${ }^{1}$, D. Yu. Ivanov ${ }^{1,2}$, A. Schäfer ${ }^{1}$, L. Szymanowski ${ }^{3,4}$ \\ ${ }^{1}$ Institut für Theoretische Physik, Universität Regensburg, D-93040 Regensburg, Germany \\ 2 Institute of Mathematics, 630090 Novosibirsk, Russia \\ ${ }^{3}$ CPhT, École Polytechnique, F-91128 Palaiseau, France* \\ 4 Soltan Institute for Nuclear Studies, Hoza 69, 00-681 Warsaw, Poland
}

(December 17, 2018)

We argue that coherent production of hard dijets by linearly polarized real photons can provide direct evidence for chirality violation in hard processes, the first measurement of the magnetic susceptibility of the quark condensate and the photon distribution amplitude. It can also serve as a sensitive probe of the generalized gluon parton distribution. Numerical calculations are presented for HERA kinematics.

PACS numbers: 12.38.-t, 11.30.Rd, 13.60.-r

The QCD vacuum is a highly complex state. It has a nontrivial particle and energy density characterized by quark and gluon condensates and complicated dynamical properties that characterize its response to external probes. In particular, consider quarks and antiquarks in the $\mathrm{QCD}$ vacuum in a constant (electro)magnetic field [1]. In the weak field, the induced magnetization of the vacuum is proportional to the applied field, the quark density $\langle\bar{q} q\rangle$, the quark electric charge $e_{q}$ and a constant $\chi$ that is called magnetic susceptibility. In relativistic notation

$$
\left\langle 0\left|\bar{q} \sigma_{\alpha \beta} q\right| 0\right\rangle_{F}=e_{q} \chi\langle\bar{q} q\rangle F_{\alpha \beta}
$$

where $F_{\alpha \beta}$ denotes the electromagnetic field strength. If the magnetic field is varying with a certain frequency, the magnetic susceptibility is replaced by the corresponding response function which is nothing else but the photon distribution function in the infinite momentum frame.

To be more precise, the wave function of a real photon contains both the perturbative chiral-even (CE) contribution of the quark-antiquark pair with opposite helicities, and the nonperturbative chiral-odd (CO) contribution with quarks having the same helicity and which is due to the chiral symmetry breaking. The perturbative CE contribution is singular $\sim 1 /|\mathbf{r}|$ at small transverse distances $\mathbf{r}$ and well known:

$$
\begin{aligned}
& \left\langle 0\left|\bar{q}(0) \gamma_{+} \frac{1 \pm \gamma_{5}}{2} q(x)\right| \gamma^{(\lambda)}(q)\right\rangle= \\
& =\frac{i N_{c} e_{q}}{4 \pi^{2} \mathbf{r}^{2}} q_{+} \int_{0}^{1} d u e^{-i u(q x)}\left[\left(e^{(\lambda)} \cdot \mathbf{r}\right)(2 u-1) \pm i \epsilon_{i k} \mathbf{r}_{i} e_{k}^{(\lambda)}\right]
\end{aligned}
$$

\footnotetext{
${ }^{*}$ Unité mixte C7644 du CNRS
}

where $\epsilon_{i k}=\epsilon_{i k+-}$ is the two-dimensional antisymmetric tensor in the transverse plane, $e_{u}=(2 / 3) \sqrt{4 \pi \alpha_{E M}}$, $e_{d}=-(1 / 3) \sqrt{4 \pi \alpha_{E M}}$, etc. The nonperturbative CO contribution is regular at small transverse separations (apart from the logarithms) and can be parametrized by the photon distribution amplitude $\phi_{\gamma}(u, \mu)$ [2]

$$
\begin{aligned}
& \left\langle 0\left|\bar{q}(0) \sigma_{\alpha \beta} q(x)\right| \gamma^{(\lambda)}(q)\right\rangle= \\
& =i e_{q} \chi\langle\bar{q} q\rangle\left(e_{\alpha}^{(\lambda)} q_{\beta}-e_{\beta}^{(\lambda)} q_{\alpha}\right) \int_{0}^{1} d u e^{-i u(q x)} \phi_{\gamma}(u, \mu) .
\end{aligned}
$$

Here the normalization is chosen as $\int d u \phi_{\gamma}(u)=1$, and $u$ stands for the momentum fraction carried by the quark.

The distribution amplitude $\phi_{\gamma}(u, \mu \geq 1 \mathrm{GeV})$ is believed to be not far from the asymptotic form

$$
\phi_{\gamma}^{\text {as }}(u)=6 u(1-u) .
$$

The magnetic susceptibility was estimated using the vector dominance approximation and QCD sum rules [3,4]:

$$
\chi\langle\bar{q} q\rangle \simeq 40-70 \mathrm{MeV} \quad(\text { at } \mu=1 \mathrm{GeV}) .
$$

However, any direct experimental evidence on both $\chi$ and $\phi_{\gamma}(u)$ is absent. The $\mathrm{CO}$ contribution in photoproduction was discussed only once in [5] for the vector meson production at large $t$.

In this letter we argue that this structure can be studied in experiments for the exclusive hard dijet production off nucleons (and nuclei)

$$
\gamma+N \rightarrow(\bar{q} q)+N
$$

similar to the recent studies of coherent dijet production by incident pions by the E791 collaboration [6]. In particular, we will show that perturbative (chirality conserving) and nonperturbative (chirality violating) contributions can be separated by the different dependence on the longitudinal momentum of the dijets and on the azimuthal angle.

The approach of [6] is different as compared to to earlier studies of the dijet photoproduction [7] in that the exclusive dijet final state is identified by requiring that the jet transverse momenta are compensated to a high accuracy within the diffractive cone and making some additional cuts. This approach seems to work for the case of coherent dijet production from nuclei by incident 
pions, and for photoproduction the corresponding experimental program is under way at HERA [8]. We assume that separation of the exclusive $q \bar{q}$ dijet final state is feasible.

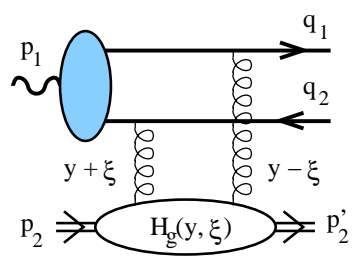

a)

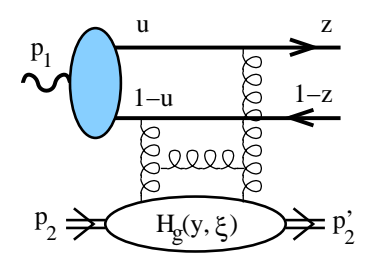

b)
FIG. 1. Sample diagrams for the hard dijet photoproduction, see text.

The kinematics of the process and the notation for the momenta is shown in Fig. 1. The Sudakov decomposition of the jet momenta with respect to the momenta of the incoming particles $p_{1}$ and $p_{2}$ reads

$$
q_{1}=z p_{1}+\frac{q_{\perp}^{2}}{z s} p_{2}+q_{\perp}, q_{2}=\bar{z} p_{1}+\frac{q_{\perp}^{2}}{\bar{z} s} p_{2}-q_{\perp}
$$

so that $z$ is the longitudinal momentum fraction and $q_{\perp}$ the transverse momentum of the quark jet. Hereafter we use the shorthand notation $\bar{u} \equiv(1-u)$ for any longitudinal momentum fraction $u$. Note that we consider the forward limit, when transverse momenta of the jets compensate each other. In this kinematics the invariant mass of the produced $q \bar{q}$ pair is equal to $M^{2}=q_{\perp}^{2} / z \bar{z}$, and the momentum of the outgoing nucleon $p_{2}^{\prime}=p_{2}(1-\xi) /(1+\xi)$, where $\xi=M^{2} /\left(2 s-M^{2}\right) \simeq M^{2} / 2 s, s=\left(p_{1}+p_{2}\right)^{2}$.

Since the CE and CO contributions lead to final states with different helicity, they do not interfere and the dijet cross section is given by the incoherent sum, for the linearly polarized photon

$$
\begin{aligned}
& \left.2 \pi \frac{d \sigma_{\gamma \rightarrow 2 \text { jets }}}{d \phi d q_{\perp}^{2} d t d z}\right|_{t=0}=\sum_{q} e_{q}^{2} \frac{\alpha_{E M} \alpha_{s}^{2} \pi^{2}(1+\xi)^{2}}{2 N_{c} q_{\perp}^{6}} \\
& \times\left[\left(1-4 z \bar{z} \cos ^{2} \phi\right)\left|\mathcal{J}_{C E}\right|^{2}+\frac{\pi^{2} \alpha_{s}^{2} \chi^{2}\langle\bar{q} q\rangle^{2}}{N_{c}^{2} q_{\perp}^{2}}\left|\mathcal{J}_{C O}\right|^{2}\right],
\end{aligned}
$$

where $\phi$ is the azimuthal angle between the jet direction and the photon polarization $\left(e^{(\lambda)} \cdot q_{\perp}\right) \sim \cos \phi, \mathcal{J}_{C E}$ and $\mathcal{J}_{C E}$ are the $\mathrm{CE}$ and $\mathrm{CO}$ amplitudes, respectively, and the prefactors are introduced for future convenience. Note that the CE contribution is $\sim 1 / q_{\perp}^{6}$ [9] and the $\mathrm{CO}$ contribution is suppressed by one extra power of $q_{\perp}^{2}$ which follows from twist counting. The different $\phi$ dependence can be traced to the fact that the $q \bar{q}$ pair is produced in a state with orbital angular momentum $L_{z}=0$ and $L_{z}= \pm 1$ for the $\mathrm{CO}$ and CE contributions, respectively.

The CE contribution originates from the region of large momenta flowing through the photon vertex and was considered previously in 10 13 in the high energy limit using $k_{\perp}$ factorization. We believe that the collinear factorization is more adequate for HERA energies and, in difference to the dijet production by incident pions [14], expect that it is valid for the $\mathrm{CE}$ amplitude to all orders in perturbation theory. Hence the amplitude $\mathcal{J}_{C E}$ is given by the convolution integral of the coefficient function and the generalized parton distribution, cf. [14]. For high energies the gluon contribution is dominant. To leading order (LO) in the strong coupling $\alpha_{s}=\alpha_{s}\left(q_{\perp}\right)$ the amplitude is given by the sum of Feynman diagrams of the type shown in Fig. 1 1 a with all possible attachments of the gluons. The answer reads

$$
\mathcal{J}_{C E}^{L O}=-\frac{1}{\pi} \int_{-1}^{1} d y \mathcal{H}_{g}(y, \xi) \frac{\xi}{(y-\xi+i \epsilon)^{2}},
$$

where $\mathcal{H}_{g}(y, \xi)=\mathcal{H}_{g}(-y, \xi)$ is the generalized gluon distribution in the symmetric notation [15], $y+\xi$ and $y-\xi$ are the $t$-channel gluon momentum fractions with respect to $\left(p_{2}+p_{2}^{\prime}\right) / 2$. The full calculation of the NLO contribution goes beyond the tasks of this letter. For the reasons explained below it is necessary, however, to include the leading NLO contribution at large energies (enhanced by $\log \xi$ ) which corresponds to an additional gluon exchange between the $t$-channel gluons, see Fig. 1 b. Including this contribution, the imaginary part of the amplitude equals

$$
\operatorname{Im} \mathcal{J}_{C E}=\xi \mathcal{H}_{g}^{\prime}(\xi, \xi)+\frac{\alpha_{s} N_{c}}{\pi} \int_{\xi}^{1} \frac{d y}{y+\xi} \mathcal{H}_{g}(y, \xi),
$$

where $\mathcal{H}_{g}^{\prime}(\xi, \xi)=d \mathcal{H}_{g}(y, \xi) /\left.d y\right|_{y=\xi}$. The real part is smaller and can be neglected in the first approximation.

For high energies alias $y \rightarrow 0, \mathcal{H}_{g}(y, \xi) \sim y^{-\Delta}$, where in perturbation theory $\Delta \sim \alpha_{s} \log s / q_{\perp}^{2}$ has to be treated as a small parameter. Therefore, despite the fact that the two terms in (10) appear in different orders in the collinear expansion, they are of the same order as far as the counting of energy logarithms is concerned. This feature is specific for real photons and can be traced to the fact that the LO amplitude in (9) only contains a (rather unusual) double-pole, but no single poles (c.f. (11)). Since $\mathcal{H}_{g}(y, \xi) \sim G(y)$ at $y \gg \xi$, and as the factor $\alpha_{s} N_{c} /(\pi y)$ is nothing but the low- $y$ limit of the DGLAP gluon splitting function, the integral in Eq. (10) can be identified to logarithmic accuracy with the unintegrated gluon distribution $f\left(\xi, q^{2}\right)=\partial G\left(\xi, q^{2}\right) / \partial \ln q^{2}$. This contribution corresponds to the one considered in $10-12]$ in the $k_{\perp}$ factorization approach. The first contribution in (10) is analogous to Eq. (42) in [13].

For the nonperturbative $\mathrm{CO}$ contribution the large momenta are not allowed in the photon vertex and the factorization formula contains a convolution with the photon distribution amplitude. In this case an additional hard gluon exchange is mandatory and the diagram in Fig. 11b presents one example of the existing 31 LO contributions, cf. Fig. 11 in Ref. [14. The calculation of this contribution is similar to the case of pion diffraction dijet production considered in much detail in 14. Here we only present the final result $\left(C_{F}=\left(N_{c}^{2}-1\right) /\left(2 N_{c}\right)\right)$ : 


$$
\begin{aligned}
& \mathcal{J}_{C O}=-\frac{1}{\pi} \int_{-1}^{1} d y \int_{0}^{1} d u \mathcal{H}_{g}(y, \xi) \frac{\phi_{\gamma}(u)}{u \bar{u}}\{ \\
& C_{F}\left(\frac{2 \xi}{(y-\xi+i \epsilon)^{2}}-\frac{1}{y-\xi+i \epsilon}\right)+\left(C_{F}\left(\frac{z \bar{z}}{u \bar{u}}+1\right)\right. \\
& \left.+\frac{1}{2 N_{c}}\left(\frac{z}{u}+\frac{\bar{z}}{\bar{u}}\right)\right) \frac{z \bar{u}+u \bar{z}}{y(z-u)-\xi(z \bar{u}+u \bar{z})+i \epsilon} \\
& \left.-\left(C_{F} \frac{z \bar{z}}{u \bar{u}}+\frac{1}{2 N_{c}}\left(\frac{z}{u}+\frac{\bar{z}}{\bar{u}}\right)\right) \frac{1}{(y-\xi-i \epsilon)}\right\}
\end{aligned}
$$

Integration over the quark longitudinal momentum fraction contains a logarithmic divergence at the end points $u \rightarrow 0,1$ which signals that the collinear factorization is violated. The divergent contribution is purely imaginary and reads

$$
\mathcal{J}_{C O}^{I R}=2 i \mathcal{H}_{g}(\xi, \xi)\left(N_{c} z \bar{z}+\frac{z^{2}+\bar{z}^{2}}{2 N_{c}}\right) \int_{u_{\min }}^{1} \frac{d u}{u^{2}} \phi_{\gamma}(u)
$$

where we have introduced an infrared cutoff $u_{\text {min }}=$ $\mu_{\mathrm{IR}}^{2} / q_{\perp}^{2}$. In numerical calculations we use $\mu_{\mathrm{IR}}=$ $500 \mathrm{MeV}$. The origin of the factorization breaking is that both final and initial state interactions are present and lead to pinching of the integration contour in the so-called Glauber region, see [14] for a detailed discussion. In the present context violation of factorization is probably not surprising since the $\mathrm{CO}$ contribution is suppressed by a power of $q_{\perp}^{2}$ compared to the leading twist.

Another important integration region for the quark momentum fraction in (11) is $\xi \ll|u-z| \ll 1$ which produces a logarithmic enhancement in energy:

$$
\mathcal{J}_{C O}^{D L A}=4 i N_{c} \phi_{\gamma}(z) \int_{\xi}^{1} \frac{d y}{y+\xi} \mathcal{H}_{g}(y, \xi) .
$$

Hence in the double-logarithmic approximation collinear factorization is valid for the $\mathrm{CO}$ contribution as well.

Assuming that the photon distribution amplitude $\phi_{\gamma}\left(z, \mu=q_{\perp}\right)$ is close to the asymptotic form (4), we obtain $\mathcal{J}_{C O} \sim z(1-z)$ for both integration regions, up to small corrections. The IR divergence in (12) does not have, therefore, any significant effect on the jet distribution but mainly influences the normalization.

In the numerical calculation presented below we have taken into account the full result in (10) and the imaginary part of the $\mathrm{CO}$ contribution in (11). Calculations are done for HERA kinematics, $s=10000 \mathrm{GeV}^{2}$ and the value $\chi\langle\bar{q} q\rangle=50 \mathrm{MeV}$, cf. (5). We use the parametrization of the generalized gluon distribution by Freund and McDermott [16] that is based on the MRST2001 leadingorder forward distribution [17]. The transverse momentum dependence of the cross section integrated over $\phi, z$ and $t$ [18] is shown in Fig. 28.

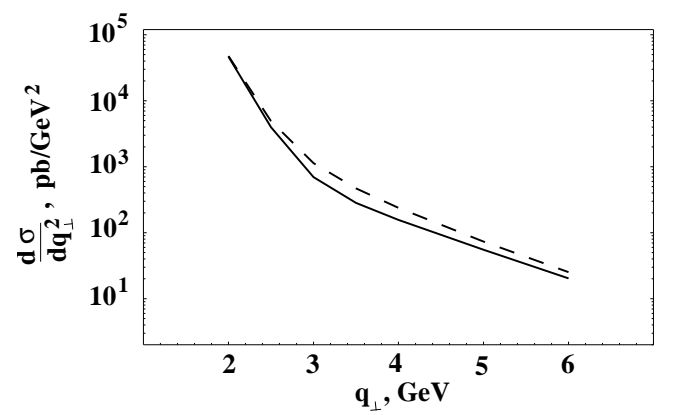

FIG. 2. The cross section $d \sigma_{\gamma+N \rightarrow 2 \text { jets }+N} / d q_{\perp}^{2}$, see text. The dashed curve corresponds to the calculation with the lower limit in the integral in (10) changed to $2 \xi$ and a different IR-cutoff $\mu_{\mathrm{IR}}=350 \mathrm{MeV}$.

For $q_{\perp}>4 \mathrm{GeV}$ the cross section is dominated by the perturbative $\mathrm{CE}$ contribution. The nonperturbative $\mathrm{CO}$ contribution at $q_{\perp} \sim 4-6 \mathrm{GeV}$ is of the order of

$$
\frac{d \sigma_{C O} / d q_{\perp}^{2}}{d \sigma_{C E} / d q_{\perp}^{2}} \simeq(7 \pm 2 \mathrm{GeV})^{2} \cdot \frac{\alpha_{s}\left(q_{\perp}\right)^{2}}{q_{\perp}^{2}}\left(\frac{\chi\langle\bar{q} q\rangle}{50 \mathrm{MeV}}\right)_{(14)}^{2}
$$

Note a large mass scale which is due to the kinematical enhancement of the $\mathrm{CO}$ amplitude by a large factor $4 \pi^{2}$, compare (2) and (3). On top of this, the two contributions in (10) have opposite sign and tend to cancel. For large transverse momenta the first term dominates, which is natural since it is leading order in the collinear expansion. However, progressing towards lower $q_{\perp}$ one effectively goes over to smaller values of Bjorken $x \sim 2 \xi$ so that the second contribution eventually becomes larger than the first one and the imaginary part of CE amplitude changes sign. This explains an abrupt change of the slope of the cross section at $q_{\perp} \sim 3 \mathrm{GeV}$. For smaller transverse momenta the dijet cross section is dominated by the $\mathrm{CO}$ contribution. Because of the cancellations in the $\mathrm{CE}$ contribution at $q_{\perp} \sim 2.5-3.5 \mathrm{GeV}$ the complete $\mathrm{NLO}$ calculation is required to make the predictions in this region fully quantitative. As a crude estimate of the uncertainties we show as dashed curve in Fig. 2 the results if the lower limit in the integral in $(10)$ is changed to $2 \xi$ and with a different IR-cutoff $\mu_{\mathrm{IR}}=350 \mathrm{MeV}$.

The transition between the two different regimes is seen very clearly from the dependence of the cross section on the dijet longitudinal momentum fraction and the azimuthal angle, compare Fig. 3 and Fig. 1 . At $q_{\perp}=5 \mathrm{GeV}$ the (parton level) $z$-distribution is almost flat, while the $\phi$ distribution is almost purely $\sim 1-\cos ^{2} \phi$. In contrast to this, at $q_{\perp}=2 \mathrm{GeV}$ the $z$-distribution is comparable with $\sim z^{2}(1-z)^{2}$ while the $\phi$-distribution is flat.

To avoid misunderstanding, we repeat that all calculations in this paper are done for the fixed value $\chi\langle\bar{q} q\rangle=$ $50 \mathrm{MeV}$ and one particular model [16] of the generalized gluon distribution. The related uncertainties are not included. In fact, coherent photoproduction of dijets may present the best opportunity to constrain both of them. 

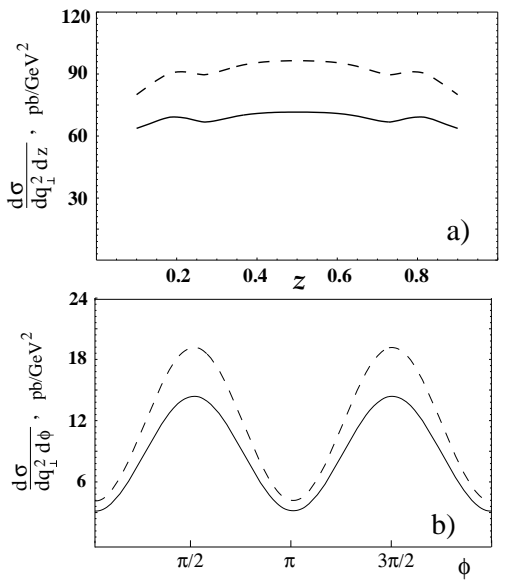

FIG. 3. The differential cross section $d \sigma_{\gamma+N \rightarrow 2 \text { jets }+N} / d q_{\perp}^{2} d z$ (a) and , $d \sigma_{\gamma+N \rightarrow 2 \text { jets }+N} / d q_{\perp}^{2} d \phi(\mathrm{b})$ for jet transverse momentum $q_{\perp}=5 \mathrm{GeV}$. Identification of the curves is the same as in Fig. 2 .
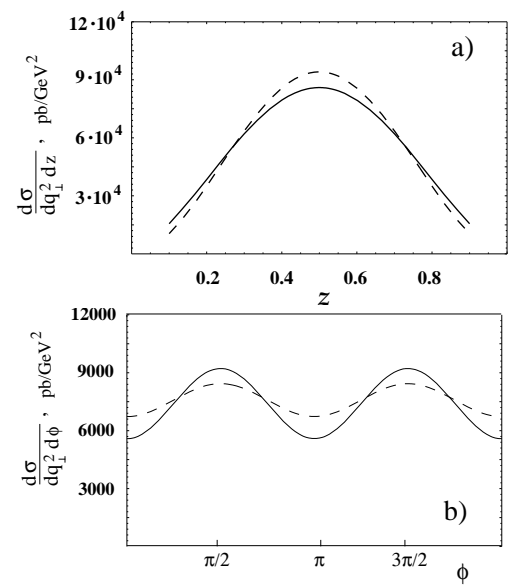

FIG. 4. Same as in Fig. 3 but for $q_{\perp}=2 \mathrm{GeV}$.

To conclude, we summarize our main points. In this letter we argue that studies of exclusive photoproduction of dijets with large transverse momenta can yield important information on the photon structure at small distances. Our main result is that the nonperturbative $\mathrm{CO}$ contribution is large in the region of intermediate $q_{\perp} \sim 2-4 \mathrm{GeV}$ and can be clearly separated from the perturbative contribution by a different $z$ - and $\phi$ dependence. Observation of the CO contribution would be the first clear evidence for the chirality violation in hard processes and also provide the first direct measurement of the magnetic susceptibility of the quark condensate. On the other hand, the dijet cross section for large $q_{\perp}$ can serve to constrain the generalized gluon distribution.

On the theoretical side, we deviate from previous studies of the dijet production by consistently applying the collinear factorization in terms of generalized parton distributions. For the nonperturbative $\mathrm{CO}$ contributions the collinear factorization is, strictly speaking, broken. However, the sensitivity to the IR cutoff is relatively weak and can formally be eliminated by taking into account Sudakov-type corrections in the modified collinear factorization framework. We think that this technique is potentially more accurate and the results can be improved systematically by the calculations of higher-order corrections. In particular, the complete NLO calculation of the perturbative $\mathrm{CE}$ contribution would be very welcome because of cancellations that are discussed in the text.

Acknowledgements. D.I. thanks the Alexander von Humboldt Foundation for the financial support. The work by S.G. was funded by the DFG grant 920770 . L.S. is grateful to B. Pire for discussions and hospitality at Ecole Polytechnique.

[1] B. L. Ioffe and A. V. Smilga, Nucl. Phys. B 232, 109 (1984).

[2] I. I. Balitsky, V. M. Braun and A. V. Kolesnichenko, Nucl. Phys. B 312, 509 (1989).

[3] V. M. Belyaev and Y. I. Kogan, Yad. Fiz. 40 (1984) 1035; I. I. Balitsky, A. V. Kolesnichenko and A. V. Yung, Yad. Fiz. 41 (1985) 282.

[4] P. Ball, V. M. Braun and N. Kivel, in preparation

[5] D. Yu. Ivanov, R. Kirschner, A. Schäfer and L. Szymanowski, Phys. Lett. B 478, 101 (2000) [Erratum-ibid. B 498, 295 (2001)].

[6] E. M. Aitala et al. [E791 Collaboration], Phys. Rev. Lett. 86, 4768 (2001).

[7] C. Adloff et al. [H1 Collaboration], Eur. Phys. J. C 6, 421 (1999).

[8] D. Ashery [E791 Collaboration], arXiv:hep-ex/0205011.

[9] L. Frankfurt, G. A. Miller and M. Strikman, Phys. Lett. B 304 (1993) 1.

[10] N. N. Nikolaev and B. G. Zakharov, Phys. Lett. B 332, 177 (1994).

[11] J. Bartels, H. Lotter and M. Wusthoff, Phys. Lett. B 379, 239 (1996) [Erratum-ibid. B 382, 449 (1996)].

[12] E. M. Levin, A. D. Martin, M. G. Ryskin and T. Teubner, Z. Phys. C 74, 671 (1997).

[13] K. Golec-Biernat, J. Kwiecinski and A. D. Martin, Phys. Rev. D 58, 094001 (1998).

[14] V. M. Braun, D. Yu. Ivanov, A. Schäfer and L. Szymanowski, Phys. Lett. B 509, 43 (2001); arXiv:hep$\mathrm{ph} / 0204191$.

[15] X. D. Ji, Phys. Rev. D 55, 7114 (1997).

[16] A. Freund and M. McDermott, Phys. Rev. D 65, 074008 (2002); http://durpdg.dur.ac.uk/hepdata/dvcs.html

[17] A. D. Martin, R. G. Roberts, W. J. Stirling and R. S. Thorne, arXiv:hep-ph/0201127.

[18] For the $t$-integration we assume $d \sigma / d t \sim \exp (b t)$ with the slope $b=6 \mathrm{GeV}^{2}$. 